\title{
Serological and haematological profile for early diagnosis of dengue infection in tertiary care hospital
}

\author{
Patel Bhavikakumari $C^{1}$, Patel Disha $A^{2, *}$, Vegad Mahendra $\mathbf{M}^{3}$ \\ ${ }^{1} 3^{\text {rd }}$ Year Resident, ${ }^{2}$ Assistant Professor, ${ }^{3}$ Professor and HOD, Dept. of Microbiology, B. J. Medical College, Ahmedabad, \\ Gujarat, India
}

*Corresponding Author:

Email: drdishapatel21@yahoo.co.in

Received: $20^{\text {th }}$ March, 2018

Accepted: $10^{\text {th }}$ May, 2018

\begin{abstract}
Context: Dengue is a major public-health concern throughout tropical and subtropical regions of the world including India. All four serotypes of the dengue can produce wide range of the symptoms from mild clinical diseases to sever diseases. Dengue haemorrhagic fever and dengue shock syndrome may occur in secondary dengue infection with serotype other than primary infection. Early diagnosis is required for proper management of this disease and to reduce mortality.

Aim and Objectives: Present study was carried out to know prevalence of dengue in Gujarat, its epidemiological status and to co-relate the serological and haematological data for early diagnosis of dengue infection.

Materials and Methods: A retrospective study was carried out over a period of one year from January 2016 to December 2016. A total of 12,413 samples having signs and symptoms of dengue fever were processed for NS 1 Ag detection and IgM Ab detection test by ELISA according to their duration of illness. Laboratory tests of dengue positive parameters and its haematological profile were co-related.

Results: A total of 1430 samples (11.52\%) were positive for dengue. Males in the age group of 16yrs to 30 yrs were predominantly affected. Monsoon and post-monsoon is a most favourable season for dengue transmission. Thrombocytopenia was more consistently associated whenever IgM was detected compared to NS1 detection.

Conclusions: Early recognition and diagnosis are important for favourable outcome. NS 1 Ag detection test by ELISA can give positive results from the $1^{\text {st }}$ day of illness. Haematological profile acts as indicators for provisional and early diagnosis of dengue.
\end{abstract}

Keywords: IgM antibody, NS1 antigen, Thrombocytopenia.

\section{Introduction}

Dengue is a major public-health concern throughout tropical and subtropical regions of the world including India. ${ }^{1}$ It is a Flavivirus belonging to the family Flaviviridae. Infection is caused by four distinct serotypes of the virus DENV-1, DENV-2, DENV-3 and DENV-4. ${ }^{2}$ It is the most rapidly spreading mosquitoborne viral disease with a wide clinical spectrum and a wide variety of presentations, ranging from asymptomatic to an undifferentiated fever to the more severe forms such as severe dengue (SD) or Dengue haemorrhagic fever (DHF). ${ }^{3}$

Over the past 50 years there was 30 -fold increase in global incidence. The World Health Organization (WHO) estimates that 50-100 million dengue infections occur each year with hundreds of thousands of severe cases, including 20,000 deaths and loss of 264 disability-adjusted life years per million populations. Thus, almost half the world's population lives in countries where dengue is endemic. ${ }^{1}$

Recovery from infection provides lifelong immunity against that serotype but confers only partial and transient protection against subsequent infection by the other three serotypes. Secondary infection with a serotype different from that causing primary infection may lead to Dengue Haemorrhagic fever and Dengue Shock Syndrome which can be fatal. ${ }^{2}$
Diagnosis of dengue fever is done by either molecular method (dengue PCR) or by serology (NS1Ag or Ab detection by ELISA).The 'gold standard' tests for identification of Dengue infection (DI) are not within the reach of peripheral and even most tertiary care laboratories. Detection of NS1 antigen and dengue specific $\operatorname{IgM} / \operatorname{IgG}$ has been the mainstay of diagnosis of DI. In developing country like us, health care system has to depend upon simple and easy laboratory tests for diagnosis. It is known that early and specific diagnosis of DHF or DSS followed by supportive therapy reduces morbidity and mortality. Apart from the dengue specific parameters, platelet count is the only accessory laboratory test available in the peripheral areas that can support the diagnosis of DHF or DSS. Even in remote areas, platelet counts can be roughly estimated by microscopy. ${ }^{4}$

In this context, the present study aimed to assess the serological and haematological dynamics of patients with dengue fever in order to diagnose early, to increase the sensitivity of the screening by healthcare professionals in the most serious cases and try to identify laboratory markers that may indicate this evolution.

\section{Materials and Methods}

This is a retrospective study, carried out in a tertiary care hospital over a period of $1^{\text {st }}$ January 2016 
to $31^{\text {st }}$ December 2016. A total of 12,413 serum samples from clinically suspected dengue cases attending our tertiary care hospital, were included in the study. Blood samples were collected in plain vacuette and serum was separated by standard protocol..$^{5}$

Samples were tested for NS1 by PLATELLA ${ }^{\mathrm{TM}}$ and IgM by NIV dengue MAC ELISA test according to the duration of their illness. (If duration of illness is $\leq 5$ days, sample is tested by NS1 ELISA and if duration of illness $>$ 5days the sample is tested by NIV dengue IgM MAC ELISA test). Detailed history was taken and platelet counts, haemoglobin, haematocrit, total white blood count, were obtained. Test results of dengue specific parameters were compared against these four parameters. Hemogram profile was done in automated cell counter, CELL-DYN 3700.

\section{Results}

Out of 12,413 samples tested during $1^{\text {st }}$ January 2016 to $31^{\text {st }}$ December 2016; 1430 (11.52\%) were positive for one or both dengue parameters. Among these, 901(63.00\%) samples were positive by NS1 antigen ELISA and 529 (37.00\%) were positive by IgM antibody ELISA, which is shown in Graph 1. Males were affected more than females, male to female ratio is 2.04: 1. Young people in the age group of 16 to $30 \mathrm{yrs}$ were affected maximum. Table 1 shows gender distribution and age distribution in the dengue positive cases. Maximum cases were found in monsoon and post monsoon seasons i.e. during the month of August to December. Seasonal distribution is shown in Graph 2. As shown in table 2, NS1 Ag detection test detect dengue from the first day of the illness and maximum positivity was seen in the third day. Platelet counts were more decreased in dengue IgM positive cases than NS1 positive cases which are shown in table 3 , whereas total leukocyte count, haemoglobin and haematocrit were more affected in NS 1 positive cases than IgM positive cases. Table 4 and 5 shows haematological parameters of dengue positive cases.

Table 1: Dengue positivity by age and sex

\begin{tabular}{|l|c|c|c|}
\hline Age group & Male (\%) & Female (\%) & Total (\%) \\
\hline $0-15$ & $246(61.97 \%)$ & $151(38.03 \%)$ & $397(27.76 \%)$ \\
\hline $16-30$ & $475(68.35 \%)$ & $220(31.65 \%)$ & $695(48.60 \%)$ \\
\hline $31-45$ & $145(63.32 \%)$ & $84(36.68 \%)$ & $229(16.01 \%)$ \\
\hline $46-60$ & $53(67.09 \%)$ & $26(32.91 \%)$ & $79(5.53 \%)$ \\
\hline$>60$ & $16(53.33 \%)$ & $14(46.67 \%)$ & $30(2.10 \%)$ \\
\hline Total & $935(65.38 \%)$ & $495(34.62 \%)$ & $1430(100 \%)$ \\
\hline
\end{tabular}

Table 2: NS 1 Positivity with duration of illness

\begin{tabular}{|l|c|c|}
\hline Duration of Illness & NS1 positive samples & \% \\
\hline Day 1 & 65 & $7.21 \%$ \\
\hline Day 2 & 253 & $28.08 \%$ \\
\hline Day 3 & 315 & $34.96 \%$ \\
\hline Day 4 & 175 & $19.43 \%$ \\
\hline Day 5 & 93 & $10.32 \%$ \\
\hline Total & 901 & $100 \%$ \\
\hline
\end{tabular}

Table 3: Platelet counts of dengue positive patients

\begin{tabular}{|l|c|c|}
\hline \multicolumn{1}{|c|}{ Platelet count } & Dengue IgM (\%) & Dengue NS1 (\%) \\
\hline$<2000 /$ cumm & $17(3.21)$ & $6(0.66)$ \\
\hline 20.000 to 50.000 & $78(14.74)$ & $35(3.88)$ \\
\hline 50,000 to 75,000 & $74(13.98)$ & $56(6.21)$ \\
\hline 75,000 to $10,00,00$ & $80(15.12)$ & $54(5.99)$ \\
\hline More than 1 lakhs & $280(52.93)$ & $750(83.24)$ \\
\hline Total & 397 & 661 \\
\hline
\end{tabular}

Table 4: Total leucocyte count of dengue positive patients

\begin{tabular}{|l|c|c|}
\hline \multicolumn{1}{|c|}{ Leucocytes count/ cmm } & Dengue IgM(\%) & Dengue NS1 (\%) \\
\hline$<4000$ & $106(20.03)$ & $221(24.52)$ \\
\hline $4000-11000$ & $360(68.05)$ & $622(69.03)$ \\
\hline$>11000$ & $63(11.90)$ & $58(6.43)$ \\
\hline Total & 529 & 901 \\
\hline
\end{tabular}


Table 5: Haemoglobin percentage of patients

\begin{tabular}{|l|c|c|}
\hline \multicolumn{1}{|c|}{$\mathbf{H b}(\mathbf{g m} / \mathbf{d l})$} & Dengue IgM (\%) & Dengue NS1 (\%) \\
\hline 3.5 to 8.5 & $35(6.61)$ & $27(2.99)$ \\
\hline 8.6 to 13.5 & $298(56.33)$ & $538(59.71)$ \\
\hline 13.5 to 18.5 & $196(37.05)$ & $336(37.29)$ \\
\hline Total & 529 & 901 \\
\hline
\end{tabular}

Table 6: Haematocrit of patients

\begin{tabular}{|l|c|c|}
\hline \multicolumn{1}{|c|}{ Haematocrit } & Dengue IgM (\%) & Dengue NS1 (\%) \\
\hline$<30$ & $69(13.04)$ & $69(7.65)$ \\
\hline 30 to 40 & $222(41.96)$ & $386(42.84)$ \\
\hline$>40$ & $238(44.99)$ & $446(49.50)$ \\
\hline Total & 529 & 901 \\
\hline
\end{tabular}

\section{Graph 1}

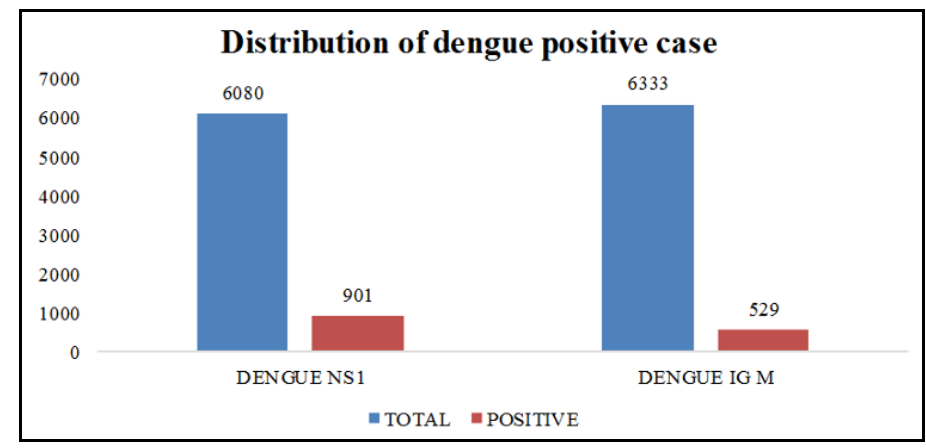

\section{Graph 2}

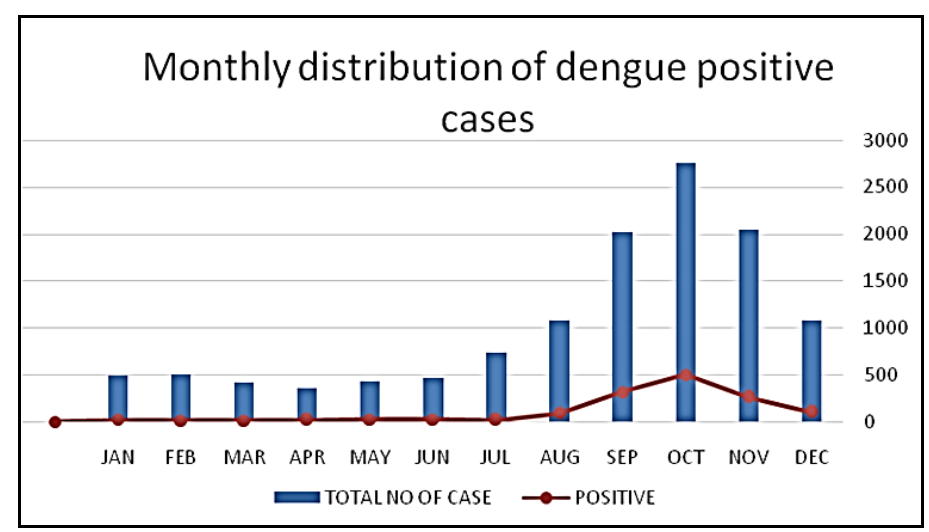

\section{Discussion}

Dengue is one of the most serious mosquito-borne viral diseases in humans. It occurs in tropical areas and affects up to 100 million people each year, with a high mortality rate in children. Infection with one of the four serotypes (1,2,3, and 4) of dengue virus may result in its classical form, a febrile illness (dengue fever [DF]). In some cases, the disease may be associated with more severe manifestations, such as haemorrhagic syndrome (dengue haemorrhagic fever) and hypovolemic shock, which often proves fatal (dengue shock syndrome). ${ }^{6}$ In order to diagnose dengue virus early, to co-relate haematological parameters with dengue serological markers and to provide timely information for the care of the patient, this study was undertaken in the
Microbiology Department of tertiary care centre during $1^{\text {st }}$ January 2016 to $31^{\text {st }}$ October 2016.

Out of total 12,413 samples, 1430 samples were found positive for dengue fever. Thus, overall seroprevalence of dengue in our study is $11.52 \%$ $(1430 / 12,413)$. Similar prevalence $(12.24 \%)$ was found in study carried out by Mehta $\mathrm{T} \mathrm{K}$ et al during 201314. ${ }^{7}$ Initial symptoms of dengue mimic those of malaria, typhoid and leptospirosis which are endemic in the country $^{8}$ so the negative cases in our study may be suffering from other causes of fever. Also samples were collected throughout the year, including period of less positivity of dengue cases though there were fewer cases in non-dengue seasons. These are the reasons of low prevalence in our study. 
Out of 12,413 samples, 6080 samples were tested by NS1 Ag ELISA test as they have duration of illness $\leq 5$ days and 6333 samples were tested by IgM ELISA test as they have duration of illness $>5$ days. Seroprevalence of dengue by NS1 Ag ELISA was 901/6080 (14.82\%) and by IgM ELISA was 529/6333 (8.35\%) (Graph 1). Out of the 1430 positive samples, $901(63.00 \%)$ were positive for NS1 \&529 (37.00\%) were positive for IgM. A study carried out by Soumya $\mathrm{K}$ et al also showed more positivity in NS1 Ag test $(49.45 \%)$ than IgM Ab test $(5.49 \%) .{ }^{2}$ Following the bite of an infected Aedes mosquito, dengue virus replicates quickly before the development of signs and symptoms. NS1 Ag circulates uniformly in all serotypes of dengue virus and circulates at high level during the 1 st few days of illness. This is the reason for high detection rate of NS1Ag in acute phase sera, there after decreases gradually and antibody detection becomes more prominent after 5 days. But practically this is not feasible as the patients never approach a clinician when there are no symptoms. ${ }^{9}$ Considering very high specificity of NS1 it can be stated that we would have missed the diagnosis in $63.00 \%$ cases if we had not included NS1 in the test panel.

In our study age group between 16 years (yrs) to $30 \mathrm{yrs}$ was affected maximum $(48.60 \%)$, followed by $15 \mathrm{yrs}$ or less than it $(27.76 \%), 31 \mathrm{yrs}$ to $45 \mathrm{yrs}(16.01 \%)$, 46 to $60 \mathrm{yrs}(5.53 \%)$ and more than 60yrs $(2.10 \%)$. Soumya $\mathrm{K}$ et al also found maximum positivity among young adults in the age group of $16-30$ years $(54.94 \%)$ to which is closely related to our study. ${ }^{2}$

As shown in table 1, gender distribution in our study shows that male $(935 / 1430-65.38 \%)$ is predominantly affected than female (495/1430 $34.62 \%)$.Male to female ratio among the seropositive cases was 1.9:1. Similar results were observed by Nerawar A et al and Atul Gerg et al who got male: female ratio 2: 1 in their study. ${ }^{8,10}$ Reasons behind male preponderance could be due to greater male exposures to dengue-carrying mosquitoes during daytime hours either at the workplace or during travelling to and from work or could be due to male-female differences in the use of health services and/or male-female differences in disease severity according to Yew et al. ${ }^{11,12}$

Graph 2 shows monthly positive cases and seasonal distribution of all dengue positive cases. It is evident from this graph that most favourable season for dengue transmission is monsoon and post monsoon as the cases starts rising from the month of the July reaches to its peak in October month and then gradually declines till the month of December. Same result was shown by study carried out by Soumya K et al. ${ }^{2}$ This is because of very favourable season for high breeding of the vector, Ades aegypti. These findings suggest that preventive measures against dengue should start well before the monsoon and continue till the end of the season. As dengue is endemic in the India very small amount of sporadic cases were also found throw-out the year.
In view of high mortality and morbidity associated with dengue especially in tropical countries, it is imperative to diagnose the disease during the early phase in order to provide information for appropriate management and avoidance of complications. NS1 is synthesized by all flaviviruses and is secreted from infected mammalian cells. ${ }^{9}$ The NS1 antigen is found together with endothelium, free or soluble in the serum of patients. The presence of secreted NS1 in the blood stream stimulates a strong humoral response and can be detected on days 0-9 after the onset of symptoms. ${ }^{9,13}$ If we see NS1 positivity with duration of illness, it is observed that NS1 antigen detection by ELISA test gives positive results from the day $1^{\text {st }}$ of the illness with maximum positivity in the day $3(34.96 \%)$ followed by day $2(28.08 \%)$, day $4(19.43 \%)$, day $5(10.33 \%)$ and day $1(7.21 \%)$. This shows that positivity starts from day one and decline gradually over the time up to $5^{\text {th }}$ day of illness. This is shown in the table 2. A study carried out by Syed I M et al also show NS1 positivity from day 1 of the illness with highest positivity in the day 3, which is well co-related with this study. ${ }^{14}$ NS 1 antigen circulates uniformly in all serotypes of dengue virus and it circulates at high level during the first few days of the illness. Antigen levels as high as 2 to 10 $\mathrm{mcg} / \mathrm{ml}$ may be found in the acute stage of the sera, while in the convalescent stage these may be 0.4 $\mathrm{mcg} / \mathrm{ml}$ or less, this is the likely explanation for the early and higher detection rate of NS1 in acute stage of the disease. ${ }^{14}$

The comparison of platelet count with dengue specific parameter is shown in table 3 . According to it, $27.97 \%$ (397/1430) dengue cases were suffered from thrombocytopenia. A study carried out by Banerjee M et al showed $19 \%$ thrombocytopenia. ${ }^{15}$ Ratagiri V H et al reported thrombocytopenia in $82 \%$; DHF in $60 \%$, DSS in $22 \%$ and DF in $18 \%$ of patients. ${ }^{16}$ Out of 529 patients positive for Dengue IgM, more than half (52.93\%) of them had platelet count more than one lacs. Similarly, more than four fifth $(85.5 \%)$ of dengue NS1 positive cases (901 cases) had platelets more than 1 lacs. Thus over all dengue IgM positive cases were more affected than NS1 Ag cases. Duthade M. M. et al also showed similar result that, Ig M positive cases (50\%) were more suffered from thrombocytopenia than NS1 $\mathrm{Ag}$ positive cases $(41 \%)$ which is well correlated with our study. ${ }^{9}$ Possibility of this could be due to duration of illness; longer the duration, higher the affection of platelet count because of progression of disease. Platelet counts are also decreased in several other conditions like some viral infections other than dengue, drug induced thrombocytopenia, collagen vascular diseases, idiopathic thrombocytopenia etc. ${ }^{17} \mathrm{We}$ therefore, tried to correlate platelet counts in cases of fever that tested negative for any of the dengue parameters \& thrombocytopenia was noted in $2818 / 10983 \quad(25.65 \%), \quad$ so association of 
thrombocytopenia with dengue parameter was significantly higher.

Association of leucocytes with dengue positive cases are shown in table 4 . Total leucocyte count results were grouped as counts $<4000,4000$ to 11000 , and $>11000 / \mathrm{cmm}$. Leucopenia (total leucocyte counts $<4000 / \mathrm{cmm})$ were seen in $327(22.87 \%)$ cases, normal count (count between 4000 to $11000 / \mathrm{cmm}$ ) were seen in $982(68.67 \%)$ cases and leucocytes $>11000 / \mathrm{cmm}$ was $21(8.46 \%)$. Ratagiri V H et al observed leucopenia in $26 \%$ of patients in their study. It is evident from the table that NS1Ag positive cases were more affected than IgM Ab positive cases. ${ }^{16}$ Myelosuppression in Dengue Fever leads to leucopenia and thrombocytopenia that occurs due to autoantibody induced phagocytosis of platelets. These laboratory data can play a complementary role in prompting the suspicion and facilitating the timely diagnosis of Dengue, even before the results of the serological tests are available. ${ }^{2}$

Haemoglobin ranged from lowest value $4 \mathrm{gm} \%$ to highest value of $19.2 \mathrm{gm} \%$ with mean haemoglobin $12.61 \mathrm{gm} \%$. Normal haemoglobin count of 8.6 to 13.5 gm\% was seen in $836(58.46 \%)$ patients, anaemia (3.5 to $8.5 \mathrm{gm} \%)$ was seen in $62(4.34 \%)$ patients and haemoglobin count of 13.5 to $18.5 \mathrm{gm} \%$ were seen in $532(37.20 \%)$ patients (table 5).Haematocrit of $>40$ was seen in $684(47.83 \%)$ patients, 30 to 40 was seen in $42.52 \%$ and $<30$ was seen in $9.65 \%$ (table 6 ) with mean haematocrit $38.86 \%$.

Based on the clinical and laboratory parameters dengue infection is divided in dengue fever and severe dengue haemorrhagic manifestation. Platelet count less than 1 lakh and haemoconcentration more than $20 \%$ quantifies for the diagnosis of DHF. In the present study thrombocytopenia was observed in $27.76 \%$ of the cases, increased haematocrit is observed in $47.83 \%$ of the cases and leucopoenia in $22.87 \%$. Haemoconcentration is an abnormality observed in this disease which is measured by increased haematocrit. Higher haematocrit is related to increased severity and is explained by the increased plasma permeability which is the basic pathophysiological alteration in Dengue. ${ }^{18}$ Thrombocytopenia is due to the direct and antibody mediated destruction of the platelets and megakaryocytes and also due to the suppression of the bone marrow by virus. Leukopenia is well established feature of dengue fever which is due to the direct marrow suppression by the virus.

Dengue fever is self-limiting disease. Dengue haemorrhagic fever causes morbidity and mortality. No antiviral treatment is available hence fluid and electrolyte replacement and supportive therapies are available modalities of treatment. Hence early and prompt diagnosis with complete hemogram helpful to give early supportive treatment to patient rather than waiting for disease to progress. Since no vaccines for this disease is available, vector control is the only way to check the transmission of the disease. ${ }^{19}$

\section{Conclusion}

Maximum prevalence of dengue is in young, in males, in rainy season with summative effect of endemicity of disease in particular geographical region. Young population bears greater burden of disease with increased magnitude in monsoon and post-monsoon period. NS 1 antigen detection test can diagnose dengue from very first day of the illness so helpful in early diagnosis of dengue. Thrombocytopenia, increased haematocrit and leucopenia are prima facie of dengue, and constitute value as indicators for provisional and early diagnosis of dengue, individually or in combination. Prompt diagnosis and immediate specific treatment with maintenance of platelet count and haemostatic function gives good recovery.

\section{References}

1. WHO: Global Strategy for Dengue Prevention and Control 2012-2020. 2012. Available from: http://www.who.int/denguecontrol/9789241504034/en/[la st assessed on 2018 February 26]

2. Kaup S, Sankarankutty J. Seroprevalence and Seasonal Trend of Dengue Virus Infection at a Teaching Hospital in Tumkur, India. Sch J App Med Sci. 2014;2(3A):92226.

3. Azin FR, Gonçalves RP, Pitombeira MH, Lima DM, Castelo Branco I. Dengue: profile of hematological and biochemical dynamics. Rev Bras Hematol Hemoter. 2012;34(1):36-41.

4. Kulkarni RD, Patil SS, Ajantha GS, Upadhya AK, Kalabhavi AS, Shubhada RM et al. Association of platelet count and serological markers of dengue infection-importance of NS1 antigen. Indian J Med Microbiol. 2011;29(4):359-62.

5. Cheesbrough M. Equipping district laboratories: Centrifuges. In District laboratory practice in tropical countries. $2^{\text {nd }}$ ed. Cambridge university press;1999.p.:138-43.

6. Alcon S, Talarmin A, Debruyne M, Falconar A, Deubel V, Flamand M. Enzyme-Linked Immunosorbent Assay Specific to Dengue Virus Type 1 Nonstructural Protein NS1 Reveals Circulation of the Antigen in the Blood during the Acute Phase of Disease in Patients Experiencing Primary or Secondary Infections. J Clin Microbiol. 2002;40(2):376-81.

7. Mehta TK and Shah PD.Evaluation of Recent WHO Classification (2009) for Assessment of Dengue Disease Severity. Int J Curr Microbiol App Sci. 2015;4(9):755-65.

8. Neralwar A, Banjare B, Barapatre R. Detection of NS1 antigen, IgM antibody for the diagnosis of dengue infection in patients with acute febrile illness. Int J Res Med Sci. 2015;3(10):2826-30.

9. Duthade MM, Damle AS, Bhakre JB, Gaikwad AA, Iravane JA, Jadhav A et al. The Study of Detection of Dengue NS1 Antigen and IgM Antibody by ELISA in and around Aurangabad, India. Int J Curr Microbiol App Sci. 2015;4(10):416-22.

10. Garg A, Garg J, Rao YK, Upadhyay GC, Sakhuja S. Prevalence of dengue among clinically suspected febrile episodes at a teaching hospital in North India. Journal of Infect Dis and Immunity. 2011;3(5):85-9. 
11. Anker M and Arima Y. Male-female differences in the number of reported incident dengue fever cases in six Asian countries. WPSAR. 2011;2(2):17-23.

12. Yew YW et al. Seroepidemiology of dengue virus infection among adults in Singapore. Annals of the Academy of Medicine, Singapore. 2009;38:667-75.

13. Solanke VN, Karmarkar MG, Mehta PR. Early dengue diagnosis: Role of rapid NS1 antigen, NS1 early ELISA, and PCR assay. Trop J Med Res. 2015;18:95-9.

14. Syed Irfan Ahmed, Ammara N, Haider ZB, Shahid IS, Zubair AR, Arshad IS et al. NS1 Antigen and Immunoglobulin $\mathrm{M}$ detection in the Acute and Early Convalescent Stages of Dengue Fever. Journal of Rawalpindi Medical College (JRMC). 2010;14(2):56-59.

15. Banerjee M, Chatterjee T, Choudhary GS, Srinivas V, Kataria VK. Dengue: A Clinicohaematological Profile. MJAFI. 2008;64:333-36.

16. Ratageri VH, Shepur TA, Wari PK, Chavan SC, Mujahid IB and Yergolkar PN. Clinical Profile and Outcome of Dengue Fever Cases. Indian J Pediatr. 2005;72(8):70506.
17. Peters CJ. Infections caused by arthropod- and Rodent borne viruses. In: Fauci AS, editor. Harrison's principles of Internal Medicine. 17 th ed. New York: McGraw- Hill Medical Publishing Division; 2008.p.1226-39.

18. Jain A, Shah AN, Patel P, Desai M, Somani S, Parikh P et al., Roopesh Singhal R, Dinesh Joshi. A clinicohematological profile of Dengue outbreak among healthcare professionals in a tertiary care hospital of Ahmedabad with analysis on economic impact. Natl J Community Med. 2013;4(2):286-90.

19. Solanki S, Bodarya O, Agnihotri OA, Joshi JR, Chokshi TS. Haematological Parameters and Their Utility in Dengue Infection. NJIRM. 2017;8(2):48-50.

How to cite this article: Patel $\mathrm{BC}$, Patel DA, Vegad MM. Serological and haematological profile for early diagnosis of dengue infection in tertiary care hospital. Indian $\mathrm{J}$ Microbiol Res. 2018;5(3):358-363. 